Check for updates

Cite this: J. Mater. Chem. A, 2019, 7, 16803

Received 10th April 2019

Accepted 13th June 2019

DOI: $10.1039 / c 9 t a 03799 c$

rsc.li/materials-a

\section{Understanding the piezoelectricity of high- performance potassium sodium niobate ceramics from diffused multi-phase coexistence and domain feature $\uparrow$}

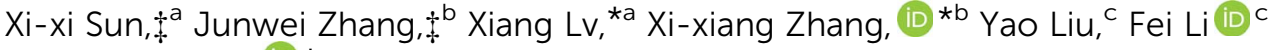 \\ and Jiagang $\mathrm{Wu} \mathbb{B D}^{* a}$
}

The understanding of high piezoelectricity in potassium sodium niobate (KNN)-based ceramics with a new phase boundary has been limited to unpoled samples. Here, the phase structure, domain structure, and phenomenological theory were studied on both unpoled and poled samples by taking $(0.99-x)\left(\mathrm{K}_{0.48} \mathrm{Na}_{0.52}\right)\left(\mathrm{Nb}_{0.955} \mathrm{Sb}_{0.045}\right) \mathrm{O}_{3}-0.01 \mathrm{SrZrO}_{3}-x\left(\mathrm{Bi}_{0.5} \mathrm{Ag}_{0.5}\right) \mathrm{ZrO}_{3}$ ceramics as an example. Shifting the phase transition temperatures to room temperature can result in the coexistence of a ferroelectric matrix containing an orthorhombic-tetragonal $(\mathrm{O}-\mathrm{T})$ coexisting phase and rhombohedral (R)-related polar nanoregions (PNRs), and then the miniature and nanoscale domain structure can be demonstrated. During the poling process, the R phase-related PNRs can facilitate domain switching and polarization rotation, resulting in a single domain structure and enhanced evidence of the $\mathrm{R}$ phase. Therefore, high piezoelectricity originates from a single domain feature as well as the diffused multi-phase coexistence in association with R phase related PNRs. This study provides a systematic approach to understand the physical mechanisms of enhanced piezoelectricity in KNN-based ceramics.

\section{Introduction}

Owing to the replacement of lead-based piezoceramics with lead-free ones, lead-free piezoceramics have been gaining popularity for more than 20 years. ${ }^{1-3}$ Potassium sodium niobate (KNN)-based ceramics are endowed with a high piezoelectric coefficient $\left(d_{33}\right)$ and moderate temperature stability due to the elaborate control of "composition design $v s$. phase structure". ${ }^{1}$ The state-of-the-art $d_{33}$ of non-textured KNN-based ceramics can reach $570 \pm 10 \mathrm{pC} / \mathrm{N}$, and is $700 \mathrm{pC} / \mathrm{N}$ for textured $\mathrm{KNN}$-based ceramics. ${ }^{4,5}$ Unfortunately, the related physical mechanisms need further exploration even if high piezoelectricity can be empirically achieved.

Both intrinsic and extrinsic contributions are considered when explaining the origin of piezoelectricity. ${ }^{6}$ The intrinsic

\footnotetext{
${ }^{a}$ Department of Materials Science, Sichuan University, Chengdu, 610065, P. R. China. E-mail:wujiagang0208@163.com; msewujg@scu.edu.cn; lvxiangscu@163.com

${ }^{b}$ Division of Physical Science and Engineering (PSE), King Abdullah University of Science and Technology (KAUST), Thuwal, 23955-6900, Saudi Arabia. E-mail: xixiang.zhang@kaust.edu.sa

${ }^{c}$ Electronic Materials Research Laboratory, Key Laboratory of the Ministry of Education, Xi'an Jiaotong University, Xi'an, 710049, P. R. China

$\dagger$ Electronic supplementary information (ESI) available. See DOI: 10.1039/c9ta03799c

\$ Xi-xi Sun and Junwei Zhang contributed equally to this work.
}

contribution mainly originates from the lattice distortion that is closely related to the phase structure, while the extrinsic contribution arises from the domain wall motion and domain switching. ${ }^{6}$ Previous publications mostly attributed the enhanced piezoelectricity of KNN-based ceramics to the multiphase coexistence and miniature domain structure. ${ }^{7}$ In addition, the grain boundary also affected the piezoelectric properties in the form of the coupling effect among grains. ${ }^{6,8}$ Such a coupling effect was closely related to the grain size of piezoceramics, i.e., the relatively large grains could promote the piezoelectric properties. ${ }^{6,8}$ The multi-phase coexistence claimed by $\mathrm{Wu}$ et al. was achieved by simultaneously shifting the rhombohedral-orthorhombic phase transition temperature $\left(T_{\mathrm{R}-\mathrm{O}}\right)$ or/and orthorhombic-tetragonal phase transition temperature $\left(T_{\mathrm{O}-\mathrm{T}}\right)$ to room temperature by doping with some indispensable additives. ${ }^{1}$ Therefore, most studies mainly focused on the relationship of composition design and piezoelectricity as well as the control ability of the additives to $T_{\mathrm{R}-\mathrm{O}}$ and $T_{\mathrm{O}-\mathrm{T}}$, but ignored how the additives affected the phase, domain and local structure of KNN-based ceramics. For example, when the same or a similar method was used to construct phase boundaries (i.e., simultaneously shifting $T_{\mathrm{R}-\mathrm{O}}$ and $T_{\mathrm{O}-\mathrm{T}}$ of $\mathrm{KNN}$ ceramics to or near room temperature), different phase structures were reported., ${ }^{9,10}$ Generally, the addition of additives would inevitably destroy the long-range 
order of $\mathrm{KNN}$ ceramics to some degree, resulting in the increased degree of diffuseness due to the occurrence of polar nanoregions (PNRs). ${ }^{11}$ Particularly, Li et al. recently revealed that PNRs played a critical role in the ultra-high piezoelectricity in PMN-PT single crystal and Sm-doped PMN-PT ceramics. ${ }^{12,13}$ However, few publications were reported to disclose the relationship between the diffuseness degree and enhanced piezoelectricity in KNN-based ceramics.

Meanwhile, the most reported domain structures were taken from the unpoled ceramics. ${ }^{14-16}$ However, the net piezoelectricity of KNN-based ceramics can only be observed by applying an external electric field that will necessarily induce domain switching and domain wall motion. ${ }^{17}$ Therefore, the observations of domain structures for both unpoled and poled samples are crucial to understand the physical origin of high piezoelectricity in KNN-based ceramics. ${ }^{18}$ Currently, chemical etching is used to observe the domain structures of poled KNNbased ceramics, ${ }^{\mathbf{1 9 2 0}}$ while this method may destroy the subtle domain structures due to the irreversible destruction. ${ }^{21}$ Recently, the ultra-fine domain structures of lead-free piezoceramics were revealed using a transmission electron microscope (TEM) with high resolution; ${ }^{15,22}$ however, the related investigations are rarely reported in $\mathrm{KNN}$-based ceramics.

Here, we chose $(0.99-x)\left(\mathrm{K}_{0.48} \mathrm{Na}_{0.52}\right)\left(\mathrm{Nb}_{0.955} \mathrm{Sb}_{0.045}\right) \mathrm{O}_{3}-$ $0.01 \mathrm{SrZrO}_{3}-x\left(\mathrm{Bi}_{0.5} \mathrm{Ag}_{0.5}\right) \mathrm{ZrO}_{3}$ (KNNS-SZ-BAZ) ceramics as an example ${ }^{23,24}$ (as described in detail in the ESI†), and the obtained piezoelectric properties further verified the success and validity of KNNS-SZ-BAZ ceramics (see Table S1 $\dagger$ ). We studied the phase structure, domain structure, and electrical properties of KNNS-SZ-BAZ ceramics, particularly focusing on how $\left(\mathrm{Bi}_{0.5} \mathrm{Ag}_{0.5}\right) \mathrm{ZrO}_{3}$ affected the phase structure, diffuseness degree and domain structure before and after the poling process. The domain structure and phase structure of the unpoled and poled KNNS-SZ-BAZ ceramics $(x=0.035)$ were respectively revealed by TEM and the temperature-dependent imaginary part of permittivity $\left(\varepsilon^{\prime \prime}-T\right)$. Based on the observed results, we systematically discussed the physical mechanisms of the enhanced piezoelectric properties. Therefore, this work contributes to understanding the physical mechanisms of the high piezoelectricity in KNN-based ceramics.

\section{Experimental procedure}

The detailed descriptions for composition design, material preparations, and characterization of phase structure, domain structure and electrical properties, as well as phenomenological theory can be found in the ESI. $\dagger$

\section{Results}

\subsection{Phase structure}

The phase structures of unpoled and poled KNNS-SZ-BAZ ceramics are analyzed in Fig. S1-S5. $\dagger$ Here, the phase diagrams of unpoled and poled KNNS-SZ-BAZ ceramics were depicted by reading $T_{\mathrm{R}-\mathrm{O}}$ from $\varepsilon^{\prime \prime}-T$ curves, and $T_{\mathrm{O}-\mathrm{T}} \& T_{\mathrm{c}}$ from both $\varepsilon^{\prime}-T$ and $\varepsilon^{\prime \prime}-T$ curves, as shown in Fig. 1(a and b). For the unpoled ceramics, $T_{\mathrm{O}-\mathrm{T}}$ monotonically reduced to or below room temperature, while $T_{\mathrm{R}-\mathrm{O}}$ increased slightly and then completely diffused to room temperature, resulting in $\mathrm{R}-\mathrm{O}-\mathrm{T}$ phase coexistence at $x=0.035$. For the poled ceramics, $T_{\mathrm{O}-\mathrm{T}}$ also monotonically reduced to or below room temperature, while $T_{\mathrm{R}-\mathrm{O}}$ almost remained unchanged at $x=0-0.035$, resulting in a nominal $\mathrm{O}-\mathrm{T}$ phase coexistence at $x=0.035$. Therefore, the discrepancy in the phase structure of the ceramics $(x=0.035)$ before and after the poling process seems to be a crucial point for understanding the origin of high piezoelectricity.

To achieve this goal, in situ Raman spectra were obtained and temperature-dependent dielectric properties were studied for the ceramics ( $x=0$ and 0.035), as shown in Fig. 2. The unpoled ceramics with $x=0$ exhibited two abnormal areas in the Raman spectra, respectively, due to the involvement of $\mathrm{R}-\mathrm{O}$ and $\mathrm{O}-\mathrm{T}$ phase transitions (Fig. 2(a)). Furthermore, the R-O phase transition exhibited a much larger zone than that of the $\mathrm{O}-\mathrm{T}$ phase transition, indicating the diffused $\mathrm{R}-\mathrm{O}$ phase transition. This was also proved in unpoled $\varepsilon^{\prime \prime}-T$ curves, in which a diffused $T_{\mathrm{R}-\mathrm{O}}$ and a sharp $T_{\mathrm{O}-\mathrm{T}}$ were observed (Fig. $2(\mathrm{~d})$ ). $\nu_{1}$ mode, representing double degenerate symmetric $\mathrm{O}-\mathrm{Nb}-\mathrm{O}$ stretching vibration, exhibited two anomalies in position with increasing temperature (Fig. 2(b)). ${ }^{25}$ The temperature points of two anomalies were consistent with $T_{\mathrm{R}-\mathrm{O}}$ and $T_{\mathrm{O}-\mathrm{T}}$ in the unpoled $\varepsilon^{\prime \prime}-T$ curve (Fig. 2(d)). The unpoled ceramics $(x=0.035)$

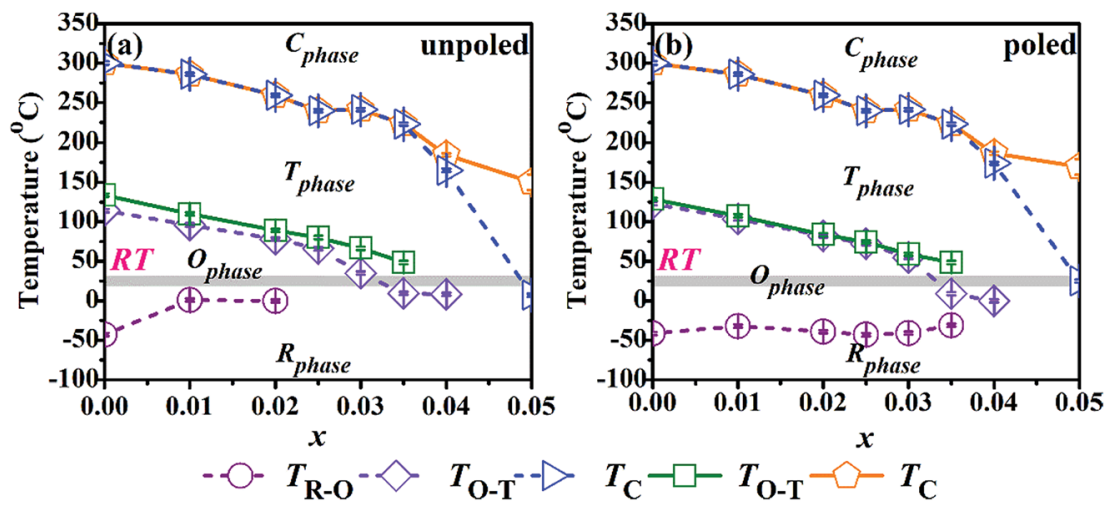

Fig. 1 Phase diagrams of (a) unpoled and (b) poled KNNS-SZ-BAZ ceramics as a function of $x$. Data of dashed and solid lines were collected from $\varepsilon^{\prime \prime}-T$ and $\varepsilon^{\prime}-T$ curves, respectively (Fig. S2 and $55 \dagger$ ). 


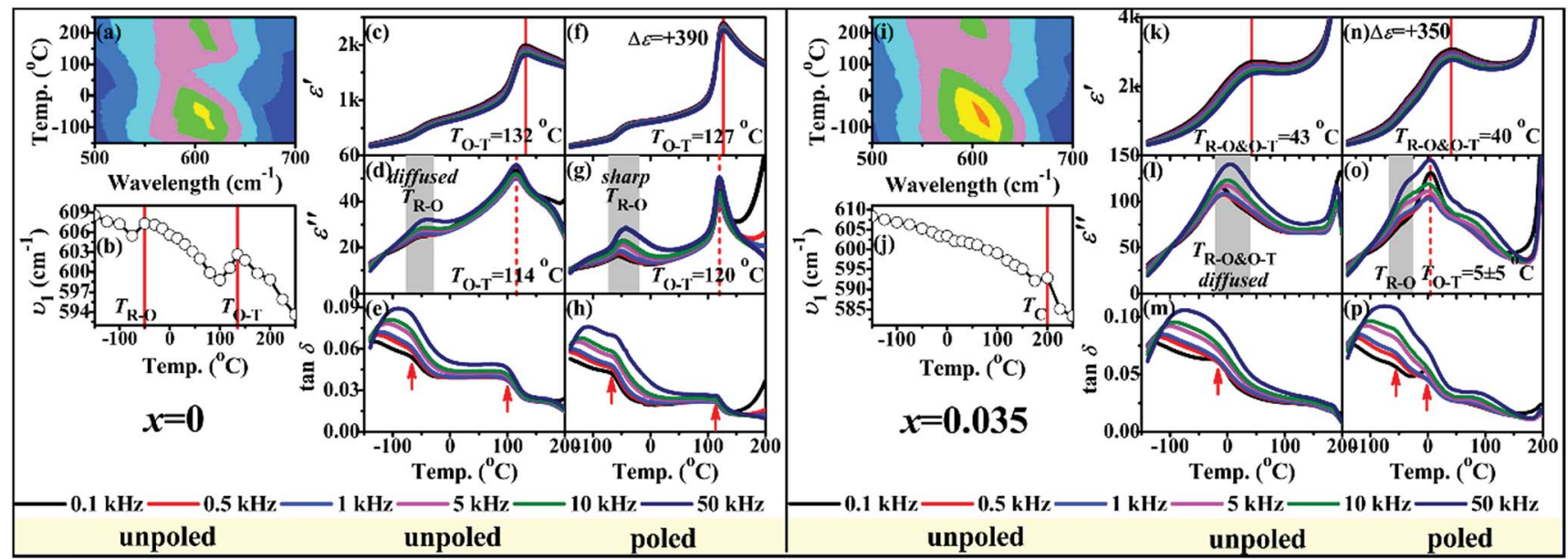

Fig. 2 In situ temperature-dependent Raman spectra of unpoled ceramics with $x=0$ (a) and $x=0.035$ (i). Raman shift of the $\nu_{1}$ mode of unpoled ceramics with $x=0$ (b) and $x=0.035$ (j). Temperature-dependent $\varepsilon^{\prime}, \varepsilon^{\prime \prime}$, and $\tan \delta$ of unpoled KNNS-SZ-BAZ ceramics with $x=0$ (c-e) and $x=$ $0.035(\mathrm{k}-\mathrm{m})$ as well as poled ones with $x=0(\mathrm{f}-\mathrm{h})$ and $x=0.035(\mathrm{n}-\mathrm{p})$. The Raman shift of the $\nu_{1}$ mode was extracted from Fig. S6. $\dagger$

also exhibited two abnormal areas in the Raman spectra (Fig. 2(i)), respectively, due to the involvement of R-O\&O-T and T-C phase transitions (Fig. 2(j)). Of particular importance was that the $\mathrm{R}-\mathrm{O}$ and $\mathrm{O}-\mathrm{T}$ phase transition zones converged into a single one, whose area was much larger than that of the R-O phase transition zone in the unpoled ceramics $(x=0)$, indicating the further increased diffuseness degree of $T_{\mathrm{R}-\mathrm{O}}$ and/or $T_{\mathrm{O}-\mathrm{T}}$. This phenomenon was further proved in the unpoled $\varepsilon^{\prime \prime}-$ $T$ curve, in which the converged $T_{\mathrm{R}-\mathrm{O}}$ and $T_{\mathrm{O}-\mathrm{T}}\left(e . g ., T_{\mathrm{R}-\mathrm{O} \& \mathrm{O}-\mathrm{T}}\right)$ was observed (Fig. 2(1)). In addition, $\varepsilon^{\prime}-T$ and $\varepsilon^{\prime \prime}-T$ curves of the unpoled ceramics exhibited different $T_{\mathrm{O}-\mathrm{T}}$ or $T_{\mathrm{R}-\mathrm{O} \& \mathrm{O}-\mathrm{T}}$ values for a given composition (e.g., $x=0$ or $x=0.035$ ) (Fig. 2(c, d, k and 1)). This discrepancy was attributed to the occurrence of PNRs and enhanced with increasing diffuseness degree. ${ }^{26,27}$ Therefore, the discrepancy of $T_{\mathrm{R}-\mathrm{O} \& \mathrm{O}-\mathrm{T}}$ in unpoled ceramics $(x=$ $0.035)$ was much larger than that of $T_{\mathrm{O}-\mathrm{T}}$ in unpoled ceramics $(x=0)$.

After the poling process, both the dielectric response and diffuseness degree were changed. The poled ceramics $(x=$ 0 and $x=0.035)$ exhibited an increase in $\varepsilon^{\prime}$ at $T_{\mathrm{O}-\mathrm{T}}$ and $T_{\mathrm{R}-\mathrm{O} \& \mathrm{O}-}$ т (Fig. 2(c, f, k and $\mathrm{n}$ )), indicating an enhanced dielectric response at phase boundaries. $\varepsilon^{\prime}-T$ curves showed reduced $T_{\mathrm{O}-\mathrm{T}}$ and $T_{\mathrm{R}-\mathrm{O} \& \mathrm{O}-\mathrm{T}}$ while the opposite changes were observed in $\varepsilon^{\prime \prime}-T$ curves, resulting in a reduced discrepancy in $T_{\mathrm{O}-\mathrm{T}}$ and $T_{\mathrm{R}-\text { O\&TO-T}}$. Meanwhile, $\tan \delta$ - $T$ curves of poled ceramics with $x$ $=0$ exhibited substantially reduced $\tan \delta$ and more sharpened anomalies at phase transitions (Fig. 2(e and h)), while the poled ceramics with $x=0.035$ only exhibited the sharpened anomalies without the reduction of $\tan \delta$. Of particular interest was that the separation of $T_{\mathrm{R}-\mathrm{O}}$ and $T_{\mathrm{O}-\mathrm{T}}$ was observed in the poled ceramics with $x=0.035$, accompanied by diffused $T_{\mathrm{R}-\mathrm{O}}$ and sharp $T_{\mathrm{O}-\mathrm{T}}$ (Fig. 2(o)). Therefore, the poling process can substantially reduce the diffuseness degree of the ceramics with $x=0$, but a decreased effect was observed for the ceramics with $x=0.035$, which was further confirmed by the variations of the diffuseness degree $(\gamma)$. After poling, $\gamma(x=0$ and $x=0.035)$ reduced from 1.32 and 1.57 to 1.19 and 1.52 , respectively (Fig. S4 and S7†).

Therefore, the discrepancy in the phase structure of the ceramics with $x=0.035$ before and after poling should be ascribed to the diffuseness of $T_{\mathrm{R}-\mathrm{O} \& \mathrm{O}-\mathrm{T}} . T_{\mathrm{O}-\mathrm{R}}$ and $T_{\mathrm{O}-\mathrm{T}}$ converged together before poling, which was the main reason why the temperature-dependent Raman spectra, $\varepsilon^{\prime \prime}-T$ curve, and Rietveld refinement revealed a diffused $\mathrm{R}+\mathrm{O}+\mathrm{T}$ phase coexistence. After poling, $T_{\mathrm{R}-\mathrm{O}}$ and $T_{\mathrm{O}-\mathrm{T}}$ were separated, resulting in a nominal $\mathrm{O}-\mathrm{T}$ phase coexistence in the $\varepsilon^{\prime \prime}-T$ curve. Here, it can be deduced that the $\mathrm{R}$ phase did exist in both unpoled and poled ceramics with $x=0.035$ in the form of a diffused phase (i.e., $\mathrm{R}$ phase related PNRs), while the ferroelectric matrix mainly possessed an $\mathrm{O}+\mathrm{T}$ coexistence phase. The status of the $\mathrm{R}$ phase was rearranged and strengthened by an external electric field, resulting in the separation of $T_{\mathrm{R}-\mathrm{O}}$ and $T_{\mathrm{O}-\mathrm{T}}$. A similar phenomenon was also reported in BCTZ ceramics. ${ }^{28}$ Brajesh et al. observed the diffused $\mathrm{R}+\mathrm{O}+\mathrm{T}$ phase coexistence in both unpoled and poled BCTZ ceramics and the increased content of the R phase in poled BCTZ ceramics by carefully analyzing the phase structure. ${ }^{28}$ However, we cannot provide more direct evidence to show the specific form of the $\mathrm{R}$ phase due to the impossibility of collecting data, which was also one of the most difficult challenges in lead-based relaxors. ${ }^{29}$

\subsection{Domain structure}

Fig. 3(a-c) show that typically large domains with a scale of $0.2-$ $1 \mu \mathrm{m}$ were observed in the unpoled ceramics with $x=0$, which were also reported in pure KNN ceramics with the O phase. ${ }^{30} \mathrm{In}$ contrast to the situation of the unpoled ceramics with $x=0$, the unpoled samples with $x=0.035$ exhibited complicated domain structures with striped, wedged, nanoscale, and irregular shapes (Fig. 3(d-h)). Striped domains with scales of 30-65 nm and 65-160 nm were observed in Fig. 3(e), and they were often reported in KNN- and lead-based ceramics with high piezoelectricity. ${ }^{151}$ Fig. $3(f)$ exhibited nanodomains with a scale of 

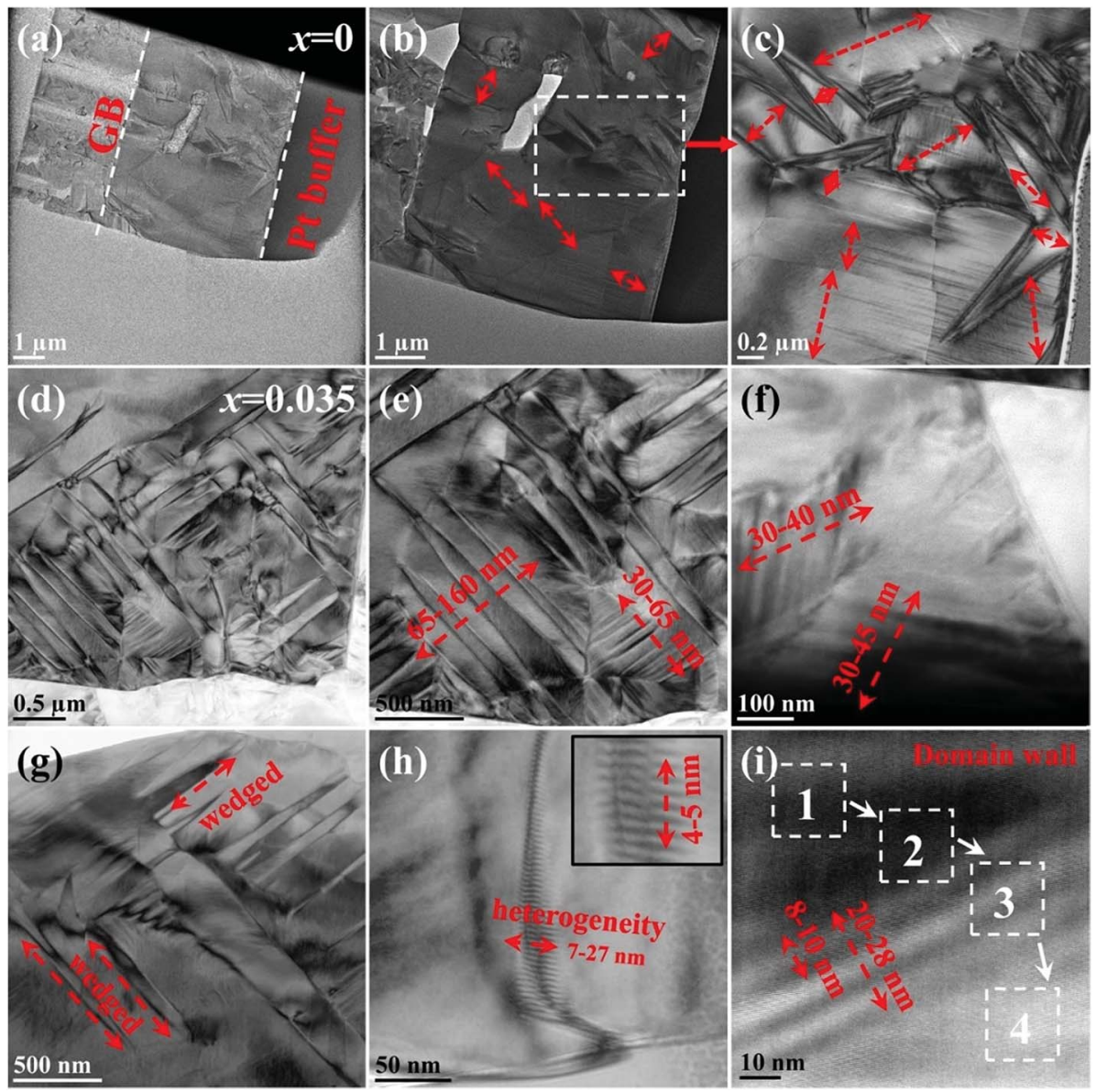

Fig. 3 Domain structures of unpoled ceramics with $x=0$ (a-c) and $x=0.035$ (d-h). (i) High-resolution TEM (HR-TEM) image of a typical domain wall in unpoled ceramics with $x=0.035$. "GB" represents the grain boundary.

30-45 $\mathrm{nm}$ parallel to each other, and they were also observed in KNNS-BNKH ceramics and believed to be responsible for the improved piezoelectricity. ${ }^{7}$ The wedged domains were considered as the typical domain patterns for rhombohedral PZT (Fig. 3(g)). ${ }^{32}$ Interestingly, ultra-fine nanodomains $4-5 \mathrm{~nm}$ in width and 7-27 $\mathrm{nm}$ in length were clearly observed in Fig. 3(h), and they exhibited an irregular distribution. These ultra-fine nanodomains were related to the local structural heterogeneity (e.g., PNRs). ${ }^{15}$ Finally, a representative domain wall separating nanoscale domains in Fig. 3(e) was enlarged to see the transition of two adjacent domain areas (Fig. 3(i)). The domain wall with a scale of 20-28 $\mathrm{nm}$ was comprised of several ultra-fine nanodomains with a scale of 8-10 $\mathrm{nm}$, indicating a gradual transition along $1 \rightarrow 2 \rightarrow 3 \rightarrow 4$, as marked by the white arrows in Fig. 3(i). Such a gradual transition was believed to decrease the domain wall energy and then reduce the total free energy. ${ }^{6}$

Fig. 4(a-f) show the domain structures of the poled ceramics with $x=0.035$. In contrast to the situation of the unpoled ceramics with $x=0.035$, the poled ceramics with $x=0.035$ exhibited simplified domain structures. The major area of the poled samples exhibited striped domains with a scale of 50$150 \mathrm{~nm}$ along the direction of the electric field (Fig. 4(b and e)). of particular importance is that a single domain zone was clearly observed in Fig. 4(c), which was also reported in BCTZ ceramics by in situ TEM and believed to be responsible for the ultrahigh piezoelectric properties. ${ }^{18}$ To further confirm the single domain structure, we deliberately cut the single domain zone into three areas with different thicknesses, forming two obvious stairs (Fig. 4(c)). In this case, the similar and continuous distribution would still be observed in a single domain zone, but not be found in a multi-domain zone, as simulated in Fig. S8. $\dagger$ A similar and continuous domain distribution was observed in this work when crossing the stairs (Fig. 4(c)), further proving the single domain zone. In the center of striped and single domain zones, an area filled with blurry domains was observed (as marked by the dashed box in Fig. 4(d)). The blurry domains were highly suspected to be related to the local heterogeneity. ${ }^{15}$ In addition, the core-shell structure was also observed, as shown in Fig. 4(f). The shell around the core exhibited wedged domains after poling (Fig. 4(f)). The simplification of the domain structure after poling was mainly due to the disappearance of $180^{\circ}$-domain walls and the reorientation of non $-180^{\circ}$ domains. ${ }^{33}$

To check the response of the domains to the electric field, out-of-plane piezoresponse force microscopy (OP-PFM) measurements were conducted on the unpoled samples with 

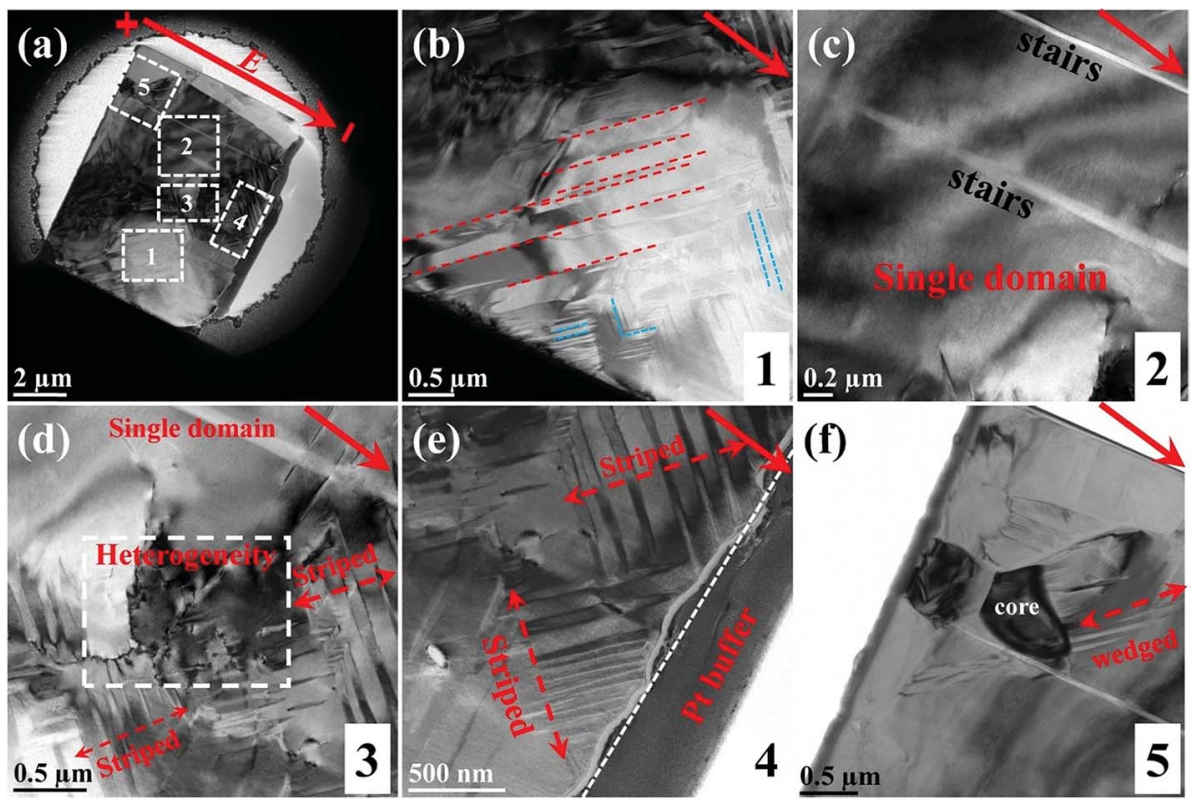

Fig. 4 (a) Poled sample of the ceramics with $x=0.035$. (b-f) Correspondingly enlarged areas in (a). The solid red arrow indicated the direction of the poling electric field.

$x=0.035$, and the results are shown in Fig. $5(\mathrm{a}-\mathrm{f})$. The amplitude of OP-PFM exhibited obvious distributions of complex domains, and the phase of OP-PFM also displayed the corresponding orientation of these domains (Fig. $5(\mathrm{a}$ and $\mathrm{b})$ ). Then, phase and piezoresponse hysteresis loop measurements were conducted (Fig. 5(c)). A typical butterfly-shaped amplitude loop and a saturated phase loop were observed when applying an AC voltage with an amplitude of $10 \mathrm{~V}$. Particularly, the phase loop exhibited the maximum phase contrast of $180^{\circ}$, indicating complete domain switching. Such a low driven voltage was half of that reported in $\mathrm{KNN}-\mathrm{BLT}-\mathrm{BZ}-\mathrm{MnO}_{2}$ and textured $\mathrm{KNN}-3 \mathrm{~T}$ ceramics, ${ }^{5,34}$ indicating easy domain switching. To further reveal the easy domain switching, writing domain measurements were conducted (Fig. 5(d and e)). The area within the white box was written by a DC voltage of $-10 \mathrm{~V}$, while the rest of the area was written by a DC voltage of $+10 \mathrm{~V}$. After writing, the whole area exhibited a strong piezoresponse with a high amplitude (Fig. 5(d)), and the phase displayed a distinct contrast in the two areas (Fig. 5(e)). Then, the statistics of the phase along the red line of Fig. 5(e) was collected and the results
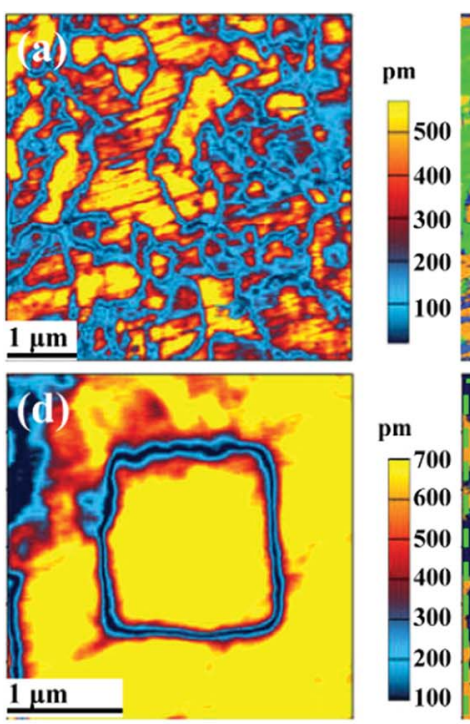
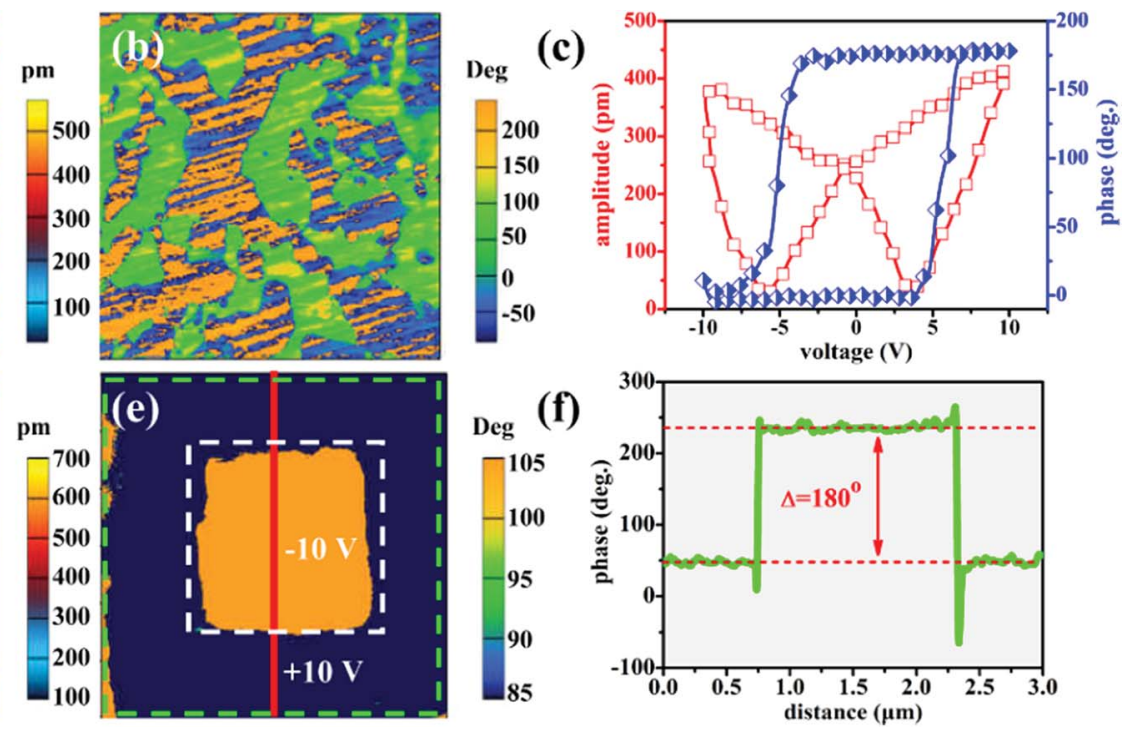

Fig. 5 (a) Amplitude, (b) phase, and (c) phase $\&$ piezoresponse hysteresis loop of out-of-plane PFM (OP-PFM) in the unpoled ceramics with $x=$ 0.035 . (d) Amplitude and (e) phase of OP-PFM in the ceramics with $x=0.035$ after writing domain measurements. (f) Phase contrast along the red line in (e). 

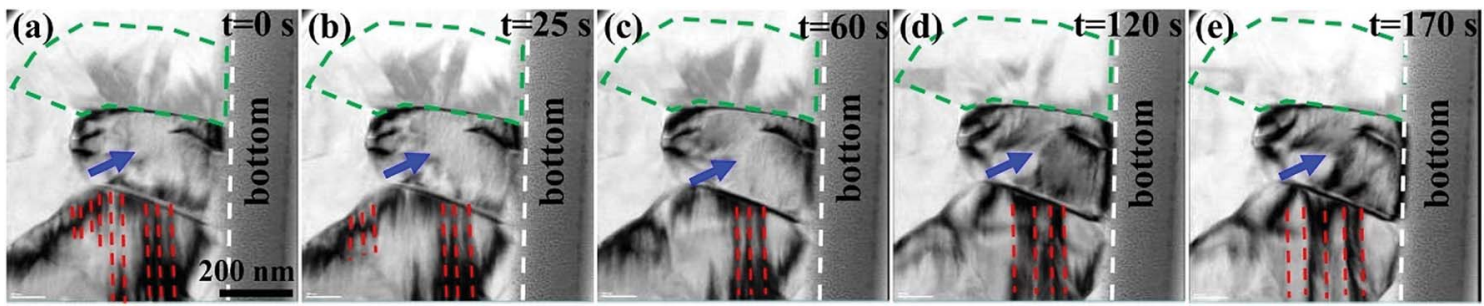

Fig. 6 (a-e) In situ TEM images of the unpoled ceramic with $x=0.035$ under exposure to an electron beam.

are shown in Fig. 5(f). A phase contrast of $180^{\circ}$ was clearly observed, indicating that a bias voltage of $\pm 10 \mathrm{~V}$ can indeed guarantee full domain switching on the surface of the unpoled sample with $x=0.035$.

The electron beam (e-beam) of TEM was reported to switch the domain structures of hexagonal yttrium manganite $\left(\mathrm{YMnO}_{3}\right){ }^{35}$ Considering the easy response of the domains to an electric field, variations of the domain structure upon exposure to an e-beam are highly expected. We thus chose an area containing the striped, irregular and nanoscale domains (Fig. 6(a)). And then, we deliberately exposed this area for near 3 minutes and took a video to show the in situ variation with time. Due to the occupation as large as $800 \mathrm{Mb}$, we thus only snapped the images at different times (Fig. 6(a-e)). As the exposure time increased, the domain walls among striped domains gradually disappeared (as marked by the green dashed line), the irregular domains became bulgy (as marked by the blue arrow), and the nanodomains grew into larger ones (as marked by the red dashed lines). These variations were mainly attributed to the electric field caused by the e-beam, indicating a fast response to an external stimulus.

\section{Discussions}

For a perovskite ferroelectric, the piezoelectric coefficient can be calculated using the equation $d_{33}=2 \alpha \varepsilon_{33} P_{\mathrm{S}}$ ( $\alpha$ is an electrostrictive coefficient, $\varepsilon_{33}$ is the dielectric permittivity and $P_{\mathrm{s}}$ represents the spontaneous polarization). ${ }^{13} \alpha$ is related to the order degree in cation arrangement, which decreased with cation order from ordered to disordered. ${ }^{36}$ The substitution of $\mathrm{Nb}^{5+}$ with $\mathrm{Zr}^{4+}$ significantly increased the disorder degree of cation order, as proved by the XRD patterns and variations of $\gamma$ (Fig. S1 and S4 $\dagger$ ), indicating reduced $\alpha$. In addition, increasing $x$ (from 0 to 0.035 ) slightly reduced $P_{\mathrm{r}}$ and $P_{\max }$, suggesting decreased $P_{\mathrm{s}}$ (Fig. S13 $\dagger$ ). Therefore, the understanding of increased $\varepsilon_{\mathrm{r}}$ (or $\left.\varepsilon^{\prime}\right)$ at room temperature plays a crucial role in unveiling the origin of high $d_{33}$ in the ceramics $(x=0.035)$.

Herein, the polar vectors of ferroelectric and PNRs were considered to explain the increased $\varepsilon^{\prime},{ }^{12}$ as shown in Fig. 7. The model was rationalized by the following reasons: (1) the ceramics with $x=0$ exhibited a larger domain size with respect to the ones with $x=0.035$ (Fig. 3); (2) the ceramics with $x=$ 0 exhibited a low degree of diffuseness before poling and a substantially reduced degree of diffuseness after poling (Fig. 2, S4 and S7 $\dagger$ ); (3) the ceramics with $x=0.035$ still exhibited a high degree of diffuseness before and after poling

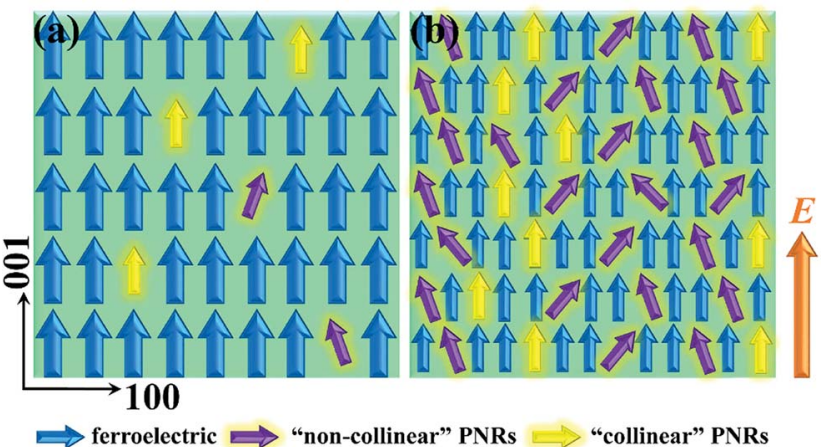

Fig. 7 Schematic diagram of polar vectors in the poled ceramics with $x=0$ (a) and $x=0.035$ (b). " $E$ " represents the applied electric field. After poling, both ferroelectric domains and PNRs were observed in the ceramics with $x=0$ and $x=0.035$. The dominating large domains with their direction along $E$ and a few PNRs were found in the ceramics with $x=0$, in which the content of "collinear" PNRs was higher than that of the "non-collinear" ones. The poled ceramics with $x=0.035$ exhibited the primary miniature domains with their direction along $E$, accompanied by an increased content of PNRs where the content of "noncollinear" PNRs was higher than that of "collinear" ones.

(Fig. 2, S4 and S7 $\dagger$ ); (4) the poled ceramics with $x=0$ exhibited a higher degree of saturation than that of the ones with $x=$ 0.035 (Fig. S9-S11†).

The ferroelectric materials with PNRs exhibit dielectric relaxation, resulting in the dependency of frequency. ${ }^{27}$ The room-temperature frequency dependence of dielectric properties in unpoled and poled samples with $x=0$ and 0.035 is discussed in Fig. S12 $\dagger$ in detail. Here, the discrepancies of $\varepsilon^{\prime}$ and its related shape in $T_{\mathrm{O}-\mathrm{T}}$ and $T_{\mathrm{c}}$ were also related to the rearranged PNRs. The part PNRs were rearranged by the electric field during the poling process, generating both "collinear" and "non-collinear" PNRs, particularly for the poled ceramics $(x=$ 0.035) (Fig. 7). Increasing the temperature to the phase transition temperatures would abruptly destroy the rearranged "collinear" PNRs, resulting in a higher $\varepsilon_{\mathrm{r}}$ (or $\varepsilon^{\prime}$ ) and a sharper dielectric anomaly (Fig. 2 and S7†).

Therefore, the high $\varepsilon_{\mathrm{r}}$ of the ceramics $(x=0.035)$ was mainly due to the existence of PNRs (particularly the "non-collinear" PNRs) and their $T_{\mathrm{R}-\mathrm{O} \& \mathrm{O}-\mathrm{T}}$ close to room temperature. $T_{\mathrm{R}-\mathrm{O} \& \mathrm{O}-\mathrm{T}}$ approaching room temperature provided a relatively high dielectric response, while the "non-collinear" PNRs could further increased the permittivity. ${ }^{12}$ That was the reason why most publications reported similarly composition-driven 
(a)
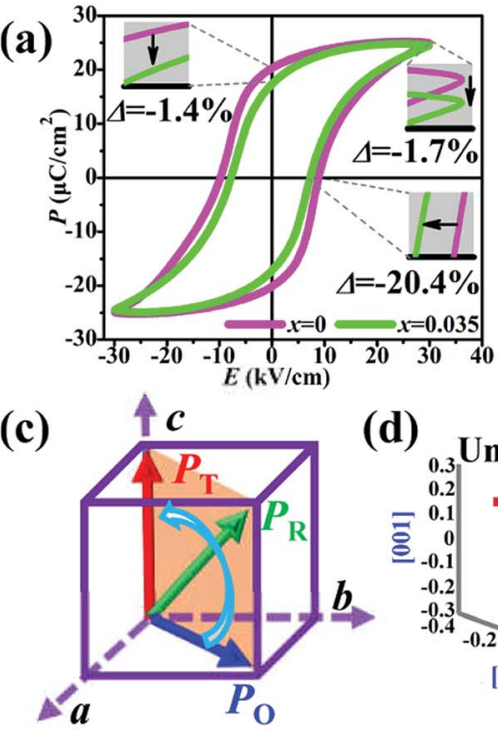

(d)

(b)

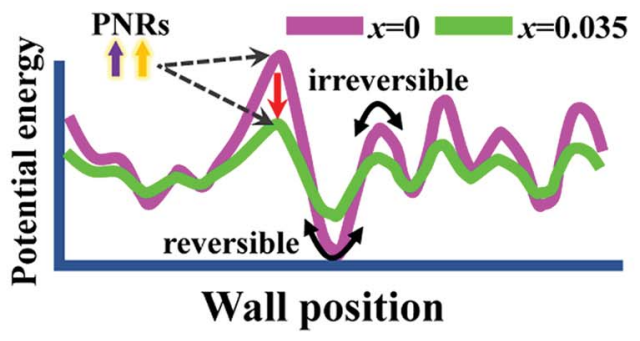

Wall position

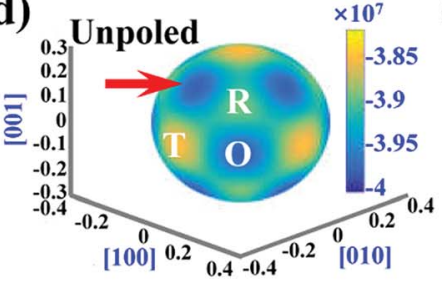

(e)

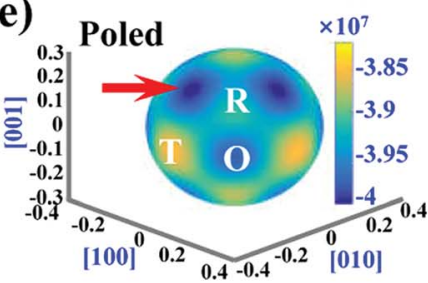

Fig. 8 (a) $P-E$ loops and (b) energy barrier for domain switching of the ceramics ( $x=0$ and $x=0.035$ ). (c) Schematic diagram of spontaneous polarization $\left(P_{\mathrm{s}}\right)$ of $\mathrm{R}, \mathrm{O}$, and T phases in KNN ceramics. Phenomenological theory simulations for (d) unpoled and (e) poled ceramics with $x=$ 0.035 .

variations of $d_{33}$ and $\varepsilon_{\mathrm{r}} P_{\mathrm{r}}$ in $\mathrm{KNN}-$, BT-, and lead-based ceramics. ${ }^{10,37,38}$ However, although the PNRs facilitated the poling process, the high content of the "non-collinear" PNRs made it difficult to achieve an ideal poling. ${ }^{12}$ This was the reason why the poled samples with $x=0.035$ exhibited a much lower phase angle with respect to the poled ones $(x=0)$ (Fig. S9 $\dagger$ ), even though much higher $d_{33}$ was found in the poled ceramics $(x=0.035)$ (Table S1 $\dagger)$. The existence of "noncollinear" PNRs may stem from the large free energy difference of the PNRs and ferroelectric matrix. ${ }^{12}$ Here, the ferroelectric matrix mainly possessed an $\mathrm{O}-\mathrm{T}$ phase coexistence, while the PNRs may exhibit an $\mathrm{R}$ phase that was strengthened and exposed after poling. Herein, a higher $d_{33}$ value is expected if one can change the "non-collinear" PNRs into the "collinear" ones as much as possible, which has been achieved in Smdoped PMN-PT ceramics but was rarely reported in lead-free ceramics. ${ }^{13}$

Since the phase boundaries of KNN-based ceramics belong to the long-range phase transition, the classical phenomenological theory needs to be considered. ${ }^{13}$ The easy domain switching was experimentally verified by OP-PFM and TEM (Fig. 3-6). Particularly, increasing $x$ from 0 to 0.035 slightly reduced $P_{\mathrm{r}}$ and $P_{\max }$, but significantly decreased $E_{\mathrm{c}}$ and the size of the domains (Fig. 3, 8(a) and S13 $\dagger$ ). The miniature domains were reported to be prone to switching under an electric field. ${ }^{39}$ The occurrence of PNRs in the ceramics with $x=0.035$ seemed to act as an "accelerator", which reduced the energy barrier and accelerated the process of domain switching (Fig. 8(b)). Herein, the extrinsic contribution for enhanced piezoelectricity can be attributed to the miniature domains and PNRs, which were easy to switch with the electric field.

Shifting $T_{\mathrm{R}-\mathrm{O}}$ and/or $T_{\mathrm{O}-\mathrm{T}}$ to room temperature not only guaranteed high $\varepsilon_{\mathrm{r}}$ but also enhanced the dielectric/ piezoelectric response of the samples to an electric field, indicating easy polarization rotation (Fig. 8(c)). To prove this, the phenomenological theory at $T=25{ }^{\circ} \mathrm{C}$ was simulated for the unpoled and poled ceramics with $x=0.035$ (Fig. 8(d and e)). Before poling, R, $\mathrm{O}$, and $\mathrm{T}$ phases exhibited a relatively flattened energy profile, indicating stable $\mathrm{R}-\mathrm{O}-\mathrm{T}$ phase coexistence and easy polarization rotation among the three phases. ${ }^{40}$ The relatively high energy of the $\mathrm{T}$ phase was due to $T_{\mathrm{R}-\mathrm{O} \& \mathrm{O}-\mathrm{T}}=43{ }^{\circ} \mathrm{C}$, which is higher than $25^{\circ} \mathrm{C}$. After poling, the energy difference of the three phases was increased (Fig. 8(e)), indicating the reduced degree of diffuseness within $\mathrm{R}-\mathrm{O}-\mathrm{T}$ phase coexistence, as proved by $\varepsilon^{\prime}-T$ and $\varepsilon^{\prime \prime}-T$ of poled ceramics $(x=0.035)$ (Fig. 2). The $\mathrm{O}$ phase exhibited the lowest energy, while the $\mathrm{T}$ phase possessed the highest energy, which was ascribed to its higher $T_{\mathrm{R}-\mathrm{O} \& \mathrm{O}-\mathrm{T}}=40{ }^{\circ} \mathrm{C}$ than $25^{\circ} \mathrm{C}$ (Fig. 2). Particularly, the energy difference of the ferroelectric matrix (with an $\mathrm{O}-\mathrm{T}$ coexistence phase) and R phase related PNRs was increased after the poling process. That was the reason why the major PNRs still existed in the "non-collinear" form in poled ceramics with $x=0.035$, even with the application of a high electric field. Therefore, the intrinsic contribution for enhanced piezoelectricity can be attributed to the diffused $\mathrm{R}-\mathrm{O}-\mathrm{T}$ coexistence phase associated with easy polarization rotation and PNR inclusions.

\section{Conclusion}

The physical mechanisms of enhanced piezoelectricity in KNNbased ceramics have been addressed. The phase structure, domain structure and phenomenological theory were studied. The addition of BAZ shifted $T_{\mathrm{R}-\mathrm{O}}$ and $T_{\mathrm{O}-\mathrm{T}}$ to room temperature, generating the phase boundary in the ceramics $(x=0.035)$ at room temperature. The unpoled ceramics $(x=0.035)$ exhibited the coexistence of the $\mathrm{O}-\mathrm{T}$ coexistence phase ferroelectric 
matrix and $\mathrm{R}$ phase related PNRs, forming a diffused $\mathrm{R}-\mathrm{O}-\mathrm{T}$ coexistence phase at room temperature. After poling, the $\mathrm{R}$ phase related PNRs were reinforced, resulting in the separation of $T_{\mathrm{R}-\mathrm{O}}$ and $T_{\mathrm{O}-\mathrm{T}}$. Due to the diffused $\mathrm{R}-\mathrm{O}-\mathrm{T}$ coexistence phase with PNR inclusions, the permittivity and the polarization rotation of the ceramics ( $x=0.035)$ were significantly improved, which contributed to enhanced piezoelectric properties as the intrinsic contribution. The unpoled ceramics $(x=0.035)$ showed complex and miniature domains, which were simplified with the help of PNRs and the electric field. Herein, the nanoscale domains and the single domain structure were observed in the poled ceramics $(x=0.035)$, acting as the extrinsic contributors.

\section{Conflicts of interest}

The authors declare no competing interests.

\section{Acknowledgements}

The authors acknowledge financial support from the National Natural Science Foundation of China (NSFC No. 517222008), the Key Technologies Research and Development Program of Sichuan Province (No. 2018JY0007), and the Undergraduate Student's Research and Innovation Fund of Sichuan University (No. 201810610075).

\section{References}

1 J. Wu, D. Xiao and J. Zhu, Potassium-Sodium Niobate LeadFree Piezoelectric Materials: Past, Present, and Future of Phase Boundaries, Chem. Rev., 2015, 115(7), 2559-2595.

2 J. Rödel, K. G. Webber, R. Dittmer, et al., Transferring leadfree piezoelectric ceramics into application, J. Eur. Ceram. Soc., 2015, 35(6), 1659-1681.

3 J. F. Li, K. Wang, F. Y. Zhu, et al., (K,Na) $\mathrm{NbO}_{3}$-Based Lead-Free Piezoceramics: Fundamental Aspects, Processing Technologies, and Remaining Challenges, J. Am. Ceram. Soc., 2013, 96(12), 3677-3696.

4 K. Xu, J. Li, X. Lv, et al., Superior Piezoelectric Properties in Potassium-Sodium Niobate Lead-Free Ceramics, $A d v$. Mater., 2016, 28(38), 8519-8523.

5 P. Li, J. Zhai, B. Shen, et al., Ultrahigh Piezoelectric Properties in Textured (K,Na) $\mathrm{NbO}_{3}$-Based Lead-Free Ceramics, Adv. Mater., 2018, 30(8), 1705171.

6 M. Acosta, N. Novak, V. Rojas, et al., BaTiO ${ }_{3}$-based piezoelectrics: Fundamentals, current status, and perspectives, Appl. Phys. Rev., 2017, 4(4), 041305.

7 T. Zheng, H. Wu, Y. Yuan, et al., The structural origin of enhanced piezoelectric performance and stability in lead free ceramics, Energy Environ. Sci., 2017, 10(2), 528-537.

8 D. K. Khatua, T. Mehrotra, A. Mishra, et al., Anomalous influence of grain size on the global structure, ferroelectric and piezoelectric response of $\mathrm{Na}_{0.5} \mathrm{Bi}_{0.5} \mathrm{TiO}_{3}$, Acta Mater., 2017, 134, 177-187.

9 X. Lv, J. Wu, S. Yang, et al., Identification of Phase Boundaries and Electrical Properties in Ternary Potassium-
Sodium Niobate-Based Ceramics, ACS Appl. Mater. Interfaces, 2016, 8(29), 18943-18953.

10 X. Wang, J. Wu, D. Xiao, et al., Giant piezoelectricity in potassium-sodium niobate lead-free ceramics, J. Am. Chem. Soc., 2014, 136(7), 2905-2910.

11 L. E. Cross, Relaxor ferroelectrics, Ferroelectrics, 2008, 76(1), 241-267.

12 F. Li, S. Zhang, T. Yang, et al., The origin of ultrahigh piezoelectricity in relaxor-ferroelectric solid solution crystals, Nat. Commun., 2016, 7, 13807.

$13 \mathrm{~F}$. Li, D. Lin, Z. Chen, et al., Ultrahigh piezoelectricity in ferroelectric ceramics by design, Nat. Mater., 2018, 17(4), 349.

14 J. Gao, Y. Hao, S. Ren, et al., Large piezoelectricity in Pb-free $0.96\left(\mathrm{~K}_{0.5} \mathrm{Na}_{0.5}\right)_{0.95} \mathrm{Li}_{0.05} \mathrm{Nb}_{0.93} \mathrm{Sb}_{0.07} \mathrm{O}_{3}-0.04 \mathrm{BaZrO}_{3}$ ceramic: A perspective from microstructure, J. Appl. Phys., 2015, 117(8), 084106.

15 Q. Liu, Y. Zhang, J. Gao, et al., High-performance lead-free piezoelectrics with local structural heterogeneity, Energy Environ. Sci., 2018, 11(12), 3531-3539.

16 Q. Liu, J.-F. Li, L. Zhao, et al., Niobate-based lead-free piezoceramics: a diffused phase transition boundary leading to temperature-insensitive high piezoelectric voltage coefficients, J. Mater. Chem. C, 2018, 6(5), 1116-1125.

17 J. Li, R. Rogan, E. Üstündag, et al., Domain switching in polycrystalline ferroelectric ceramics, Nat. Mater., 2005, 4(10), 776.

18 H. Guo, C. Zhou, X. Ren, et al., Unique single-domain state in a polycrystalline ferroelectric ceramic, Phys. Rev. B: Condens. Matter Mater. Phys., 2014, 89(10), 100104.

19 Y. Qin, J. Zhang, Y. Gao, et al., Study of domain structure of poled (K,Na) $\mathrm{NbO}_{3}$ ceramics, J. Appl. Phys., 2013, 113(20), 84.

20 Y. Qin, J. Zhang, W. Yao, et al., Domain Configuration and Thermal Stability of $\left(\mathrm{K}_{0.48} \mathrm{Na}_{0.52}\right)\left(\mathrm{Nb}_{0.96} \mathrm{Sb}_{0.04}\right) \mathrm{O}_{3}$ $\mathrm{Bi}_{0.50}\left(\mathrm{Na}_{0.82} \mathrm{~K}_{0.18}\right)_{0.50} \mathrm{ZrO}_{3}$ Piezoceramics with High $d_{33}$ Coefficient, ACS Appl. Mater. Interfaces, 2016, 8(11), 72577265.

$21 \mathrm{~J} . \mathrm{Wu}$, Advances in Lead-free Piezoelectric Materials, Springer, 2018.

22 X. Liu and X. Tan, Giant strains in non-textured $\left(\mathrm{Bi}_{1 / 2} \mathrm{Na}_{1 / 2}\right)$ $\mathrm{TiO}_{3}$-based lead-free ceramics, Adv. Mater., 2016, 28(3), 574578.

23 X. Lv, J. Wu, D. Xiao, et al., Modifying Temperature Stability of $(\mathrm{K}, \mathrm{Na}) \mathrm{NbO}_{3}$ Ceramics through Phase Boundary, Adv. Electron. Mater., 2018, 4(9), 1800205.

24 X. Wang, J. Wu, D. Xiao, et al., New Potassium-Sodium Niobate Ceramics with a Giant $d_{33}$, ACS Appl. Mater. Interfaces, 2014, 6(9), 6177-6180.

25 F. Rubio-Marcos, M. Banares, J. Romero, et al., Correlation between the piezoelectric properties and the structure of lead-free KNN-modified ceramics, studied by Raman Spectroscopy, J. Raman Spectrosc., 2011, 42(4), 639-643.

26 A. Tagantsev, Vogel-Fulcher relationship for the dielectric permittivity of relaxor ferroelectrics, Phys. Rev. Lett., 1994, 72(7), 1100. 
27 Y. Guo, K.-I. Kakimoto and H. Ohsato, Ferroelectric-relaxor behavior of $\left(\mathrm{Na}_{0.5} \mathrm{~K}_{0.5}\right) \mathrm{NbO}_{3}$-based ceramics, J. Phys. Chem. Solids, 2004, 65(11), 1831-1835.

28 K. Brajesh, K. Tanwar, M. Abebe, et al. Relaxor ferroelectricity and electric-field-driven structural transformation in the giant lead-free piezoelectric (Ba,Ca)(Ti,Zr) $\mathrm{O}_{3}$, Phys. Rev. B: Condens. Matter Mater. Phys., 2015, 92(22), 224112.

29 H. Takenaka, I. Grinberg, S. Liu, et al., Slush-like polar structures in single-crystal relaxors, Nature, 2017, 546(7658), 391.

30 R. López-Juárez, O. Novelo-Peralta, F. González-García, et al., Ferroelectric domain structure of lead-free potassiumsodium niobate ceramics, J. Eur. Ceram. Soc., 2011, 31(9), 1861-1864.

31 D. You, W. Jung, S. Choi, et al. Domain structure in a micronsized $\mathrm{PbZr}_{1-x} \mathrm{Ti}_{x} \mathrm{O}_{3}$ single crystal on a Ti substrate fabricated by hydrothermal synthesis, Appl. Phys. Lett., 2004, 84(17), 3346-3348.

32 M. Abplanalp, D. Barošová, P. Bridenbaugh, et al., Scanning force microscopy of domain structures in $\mathrm{Pb}\left(\mathrm{Zn}_{1 / 3} \mathrm{Nb}_{2 / 3}\right) \mathrm{O}_{3^{-}}$ $8 \% \mathrm{PbTiO}_{3}$ and $\mathrm{Pb}\left(\mathrm{Mg}_{1 / 3} \mathrm{Nb}_{2 / 3}\right) \mathrm{O}_{3}-29 \% \mathrm{PbTiO}_{3}$, J. Appl. Phys., 2002, 91(6), 3797-3805.

33 C. Zhou, J. Zhang, W. Yao, et al., Piezoelectric performance, phase transitions, and domain structure of $0.96\left(\mathrm{~K}_{0.48} \mathrm{Na}_{0.52}\right)\left(\mathrm{Nb}_{0.96} \mathrm{Sb}_{0.04}\right) \mathrm{O}_{3}-0.04\left(\mathrm{Bi}_{0.50} \mathrm{Na}_{0.50}\right) \mathrm{ZrO}_{3}$ ceramics, J. Appl. Phys., 2018, 124(16), 164101.
34 M.-H. Zhang, K. Wang, Y.-J. Du, et al., High and temperatureinsensitive piezoelectric strain in alkali niobate lead-free perovskite, J. Am. Chem. Soc., 2017, 139(10), 3889-3895.

35 Z. Chen, X. Wang, S. P. Ringer, et al., Manipulation of nanoscale domain switching using an electron beam with omnidirectional electric field distribution, Phys. Rev. Lett., 2016, $117(2), 027601$.

$36 \mathrm{~F} . \mathrm{Li}, \mathrm{L} . \mathrm{Jin}, \mathrm{Z}$. Xu, et al., Electrostrictive effect in ferroelectrics: An alternative approach to improve piezoelectricity, Appl. Phys. Rev., 2014, 1(1), 011103.

37 C. Zhao, H. Wang, J. Xiong, et al., Composition-driven phase boundary and electrical properties in $\left(\mathrm{Ba}_{0.94} \mathrm{Ca}_{0.06}\right)\left(\mathrm{Ti}_{1-x} \mathrm{M}_{x}\right)$ $\mathrm{O}_{3}(\mathrm{M}=\mathrm{Sn}, \mathrm{Hf}, \mathrm{Zr})$ lead-free ceramics, Dalton Trans., 2016, 45(15), 6466-6480.

38 R. Nie, Q. Zhang, Y. Yue, et al., Phase structure-electrical property relationships in $\mathrm{Pb}\left(\mathrm{Ni}_{1 / 3} \mathrm{Nb}_{2 / 3}\right) \mathrm{O}_{3}-\mathrm{Pb}(\mathrm{Zr}, \mathrm{Ti}) \mathrm{O}_{3}$ based ceramics, J. Appl. Phys., 2016, 119(12), 124111.

39 P. Marton, I. Rychetsky and J. Hlinka, Domain walls of ferroelectric $\mathrm{BaTiO}_{3}$ within the Ginzburg-LandauDevonshire phenomenological model, Phys. Rev. B: Condens. Matter Mater. Phys., 2010, 81(14), 144125.

40 C. Zhao, H. Wu, F. Li, et al., Practical High Piezoelectricity in Barium Titanate Ceramics Utilizing Multiphase Convergence with Broad Structural Flexibility, J. Am. Chem. Soc., 2018, 140(45), 15252-15260. 